that the dose received by the population in these regions has now risen to roughly twice the natural background level. External irradiation of the gonads by fallout is, in Europe and North America, very much smaller, probably I or $2 \%$ of the average dose distributed in the practice of diagnostic radiology.

(2) There is the risk of leukaemia or bone cancer through the ingestion and deposition of ${ }^{90} \mathrm{Sr}$. This hazard is very difficult to estimate. The majority opinion in scientific and medical circles is that the strontium hazard is appreciable, but not yet dangerous in comparison with other widespread sources of radiation in our own country. In the rice-eating countries of the East, the strontium hazard is very much greater* and (because X-ray services are less well developed) the hazard from diagnostic $\mathrm{X}$-rays is relatively small.

\title{
Conclusion
}

The one conclusion which emerges from the study of these problems is that we do not know enough to make any accurate estimate of the dangers to be faced from radioactive contamination of food, now or in the future. A much greater research effort is needed to tackle this difficult situation before it becomes any worse, as it will do if large hydrogen bombs continue to be exploded. More work and less talk must be the policy.

\footnotetext{
*Vegetables and cereals generally contain much more ${ }^{0} \mathrm{Sr} / \mathrm{g}$ calcium than do milk and other animal products. The intake of ${ }^{\circ} \mathrm{Sr}$ is therefore greater in countries where rice constitutes the main source of dietary calcium than in regions such as Europe and America where most of the calcium is provided by milk.
}

REFERENCE

Brown, W. M. C. (1959). Proc. Nutr. Soc. 18, 38 .

\section{Some reflections on the possible hazards to man of low doses of radiations}

By W. M. Court Brown, Medical Research Council, Group for Research into the General Effects of Radiation, Western General Hospital, Edinburgh

Before any quantitative evaluation of a hazard can be made two basic types of information are necessary: firstly, the distribution in the environment of the hazardous agent; and secondly, the biological relationship between the frequency of occurrence of any particular harmful effect and the amount of the agent present in the relevant target tissue. A considerable body of information is now available on the distribution in the environment, and in man himself, of long-life fission products derived from megaton test explosions, in particular on strontium ${ }^{89} \mathrm{Sr}$ and ${ }^{90} \mathrm{Sr}$ ) and caesium $\left({ }^{137} \mathrm{Cs}\right)$. If a similar amount of knowledge was available on the doseresponse relationships for various harmful effects of radiation we would be in a very strong position from the point of view of estimating the hazard. Unfortunately, 
human biological studies lag far behind the physical studies of distribution and biological studies in animals and plants, not only on the possible hazardous effects of fission products, but also on the effects of nuclear and allied radiations of all kinds. It is important for scientists and medical men to appreciate how little knowledge is available, and to understand the controversial aspects of dose-response relationships. This communication discusses three important biological effects of radiations: genetic effects, the effect on longevity, and the induction of tumours.

\section{Genetic effects}

For several years, it has been held that a proportional relationship exists between mutation frequency and radiation dose. The foundation of this belief has been work on Drosophila melanogaster, in which it has been claimed that for sex-linked lethals there is a linear relationship down to a dose level of $25 \mathrm{r}$ (Muller, 1954). It has been postulated, and the postulate has been generally accepted, that the same type of relationship will also hold for doses below $25 \mathrm{r}$, and for other types of mutations, particularly recessive mutations. If it is so then, firstly, the effect is one from which no recovery occurs, i.e. the induced mutations are stable and not subject to repair; secondly, there is no important amount of intercellular selection such as change in the relative frequencies of mutant and non-mutant cells within an individual's lifetime; and thirdly, radiation given at one time does not, by some long-term after-effect, influence the mutagenicity of cells irradiated at a later period. In short, it is proposed that there is a simple additive relationship, and that the frequency of the induction of mutations is independent of the time over which a given dose of radiation is received.

Satisfactory studies have yet to be made of radiation-induced genetic effects in man but, because of the universal nature of the mechanism of inheritance, the same type of relationship is believed to hold for man as for other forms of life, certainly in a qualitative sense. It is also believed that almost all radiation-induced mutations will be harmful, on the grounds that, as man has evolved in the face of a constant radiation background, virtually all the beneficial mutations will have been utilized in his evolution through the processes of natural selection.

Recently, however, the basic tenet of proportionality has been questioned, in particular by Russell (1958) of Oak Ridge. Russell has worked with male mice irradiated with X-rays at a high dose rate, and mated after recovery from the postirradiation sterile period, that is after recovery of the spermatogonia, so that there was a high probability that the mutations observed were 'point' mutations. He has found, for a number of mutations at specific loci, an appreciable departure from linearity as the dose is decreased, and that this departure takes the form of the occurrence of more mutations than would be expected on the basis of simple proportionality and the results at the higher levels of dose. He considers the most likely explanation of this important finding to be a heterogeneity amongst spermatogonia in regard to their radiation sensitivity, and that sensitivity to damage resulting in cell death is positively correlated with sensitivity to induction of mutation. Thus at higher doses more of the sensitive spermatogonia would be killed, and the mutation rate in the more resistant surviving cells would be lower. This finding, if confirmed, 
is important from the point of view of any attempt to deduce from the results in mice, the only mammal which has been really extensively studied, the genetic effects of radiation on man. It may well be that the effects of comparatively low doses given at high dose rates (e.g. diagnostic dose levels) and the effects of low doses received at low dose rates (occupational and environmental dose levels) are somewhat greater than had been expected.

\section{Radiation and life span}

In 1956 the U.S. National Academy of Sciences published a report on the effects of radiation in which were included some data on the mean age at death of American radiologists in contrast to the mean age at death of American physicians having no occupational contact with radiations ((U.S.A.) National Research Council, 1956). It was concluded that, as the mean age of death of the radiologists was some 5 years less than that of the physicians, occupational exposure might have reduced the expectancy of life. Furthermore, as in an additional report (Warren, 1956) an analysis of several major causes of death among the same subjects showed about the same discrepancy, it was assumed that the effect was non-specific; that is that radiation conferred upon the individual a degree of general unfitness to survive reflected in an increase in the age-specific mortality rates for all causes of death.

The subject is clearly important, as perhaps by the end of the present century in Britain alone, there may be upwards of half-a-million persons classified as radiation workers. Any detectable reduction in expectation of life by low doses of radiation, such as the loss of 5 days/ $\mathrm{r}$ received by the whole body, as has been suggested by Jones (1957), could amount to an appreciable economic problem, quite apart from the undesirable effect on the individuals concerned. Furthermore, the suggestion put forward by Jones was not without some foundation in fact, as animal experiments have established that large sublethal doses of radiation given at high dose rates shorten expectation of life, when the expectation is scored from the time of recovery from the acute effects of the radiation. It has been claimed that this shortening is a non-specific effect and is not due to the operation of several specific causes of death, such as tumours and renal damage. These results have also been claimed to support the theoretical contention that an important feature in the process of ageing, and presumably a basic cause, is the progressive accumulation of deleterious somatic mutations with age. It would be idle to deny that such mutations occur after radiation exposure, as it does not appear logical to accept that radiations induce mutations in germinal tissues and at the same time to deny that mutations can be produced in the stem cells of somatic tissues. In fact, Loutit and his colleagues have been able to demonstrate, in the recovery of the marrow cells of mice grafted with homologous marrow and irradiated over the whole body, clones of cells carrying what must be radiation-induced genetical damage (Loutit, 1958). The point at issue, therefore, is not whether non-specific shortening of life occurs as a result of somatic genetic damage, but whether reduction in the expectation of life can be demonstrated in persons exposed to low doses of radiations. If it cannot be detected, then whether it occurs or not becomes a purely academic question. In the light of these arguments it 
became of some importance to investigate the validity of the claim about the reduction in the expectation of life of radiologists.

Accordingly, a survey was carried out into the mortality of British radiologists during the 60 years from 1897 to 1956 , a period covering the whole development of medical radiology, so that the study population included the pioneer British radiologists, many of whom were known to have exposed themselves excessively, as can be judged by their raised mortality from skin cancer (Brown \& Doll, 1958). The results of this survey provide no evidence that there has existed any overall increase in the age-specific mortality rates such as would be expected if the process of ageing had been accelerated by exposure. There are, of course, those who argue, and probably argue quite correctly, that age-specific mortality rates are not a true measure of the rate of ageing, and that a more exact and reliable measure would be some such parameter as the elasticity of connective tissue. It is, however, a reasonable assumption that, as more exact measures are not practicable in man, the age-specific mortality rates for all causes of death, in a modern society, reflect in good measure the rate of ageing. Data have recently been published in the United States which show that the results of the analysis by Warren (1956) of the ages at death of American radiologists were due to a difference in the age-distribution of radiologists compared with that of the medical profession as a whole (Seltser \& Sartwell, I958). Radiologists, because of their specialist training, enter the practice of radiology at a somewhat later age, but because radiology is a young and expanding speciality there is a deficiency of radiologists in the older age groups in comparison with the rest of the medical profession. It is concluded, therefore, that low levels of radiation dose have no detectable effect on man's expectation of life.

\section{Induction of tumours}

It could be argued that, as far as genetic effects are concerned, strontium, though not caesium, could hardly provide any very effective irradiation of gonads; also, it appears extremely unlikely that either nuclide could, at the levels of dose received under peacetime conditions, affect the expectation of life. But the position as far as the induction of tumours is concerned remains debatable, the crux of the situation ultimately being the mechanism of tumour induction. The two types of cancer that have given rise to anxiety in relation to radioactive fallout are bone tumours and leukaemias.

It goes without saying that the probability of either form of tumour appearing is very materially increased if a sufficiently large amount of a bone-seeking nuclide is present in the body. The question at once arises of what information is available for man that supports the contention that even very small amounts of radiation may render him more liable to develop these lesions. The short and accurate reply to this question is that, for man, no information exists at all on the effects on the incidence of either bone tumours or leukaemias of very low levels of exposure to radiation. In the absence of such knowledge, assumptions have to be made on what is known about the effects of relatively high doses of radiation. 
Bone tumours. Most of the information on the induction of bone tumours comes from American studies on, firstly, persons who have been employed in the industrial application of radioactive paints and, secondly, a number of patients who have been given radium salts as a therapeutic measure. Several features complicate the interpretation of the findings in these two groups. Perhaps the most important, from the general standpoint of epidemiology, is that considerable selection has gone into the formation of both groups. The most frequent factor is that the patient was not recognized as having a body burden of radioactive nuclide until he developed signs of its effects, either areas of destruction of non-cancerous bone or frank bone tumours. Again, the size of the total population from which the subjects were drawn can never be known, and they cannot be regarded as a random sample of the total group involved. A second complication is that many patients had absorbed, not pure radium, but a radium-mesothorium mixture. Because of the differing natures of the decay schemes of these two elements, the maximum amount of energy absorbed in the body per atom decaying is very appreciably greater for mesothorium than for radium. For equivalent amounts of the elements ingested, one might therefore expect to get more lesions from mesothorium than from radium. In fact, the lowest body burden of pure radium that has so far been found associated with a tumour is about $3.6 \mu \mathrm{c}$ whereas a tumour has been found in an individual with a body burden of $0.52 \mu \mathrm{c}$ of a radium-mesothorium mixture. As far as non-malignant lesions are concerned, a dental lesion has been associated with a body burden as low as $0^{\circ} 15 \mu \mathrm{c}$ of probable radium-mesothorium mixture, and no less than thirty-two of forty-four patients having body burdens of $0.4 \mu \mathrm{c}$ or more had radiological evidence of bone damage. When it is recalled that the maximum permissible level for radium has been placed at a body burden of $O \cdot I \mu \mathrm{c}$, it is understandable that some feel that the safety margin is rather small, if indeed there is one at all. To date, as a result of comparative animal studies, the maximum permissible level for strontium has been placed at $\mathrm{r} \cdot \mathrm{o} \mu \mathrm{c}$, ten times that for radium; however, there is no real way of telling whether it is justifiable or not, and it is a matter of pure conjecture whether the present levels of strontium in the world population confer an increased probability of formation of bone tumour. In essence, the attitude towards this topic depends on what particular views are held on the mechanism of tumour induction by radiation.

Leukaemia. The controversy about the mechanism of tumour induction is accentuated on the subject of radiation leukaemogenesis. This is because some data are available on the possible nature of the relationship between the dose received by the bone marrow and the incidence of leukaemia (Brown \& Doll, 1957; Brown, 1958).

There are two widely contrasting views on this problem. One is that the tumour can result solely from damage to the genetic mechanism of a suitable cell, damage which may range from a simple point mutation to more complex chromosomal disturbances following multiple chromosomal breaks, such as inversions and translocations. The opposing view is that a definite threshold of dose must be exceeded before the tumour can develop; many proponents of this view think that this threshold may be quite high, and that the actual tumour is a manifestation of perverted repair. It may well be that there is an element of truth in both arguments, 
and that the key to the problem may be the remarkable diversity of damage that radiation can inflict on the genetic structure of the cell. In most instances such damage will ultimately cause the death of the cell. Sometimes, however, damage may result in the initiation de novo of a frankly malignant cell, possibly a cell whose products have a selective advantage over all other cells of the same type. Again, in other instances, the result may be simply to induce in the cell a pre-malignant state, the complete malignant transformation only occurring after the superimposition of the influence of some other factor. This other factor may be more gross marrow damage due to radiation or a modification of some homeostatic mechanism as a result of radiation damage elsewhere in the body. It could be that leukaemias will be but rarely induced solely as a result of genetic radiation damage, and that they are more likely to result from the combined effect of genetic radiation damage and a second factor. On this model, therefore, one would expect a non-threshold type of curvilinear dose-response curve with a shallow rising curve at very low doses succeeded by a more steeply rising curve as the dose increases and, logically, a terminal flattening of the curve as the dose becomes great enough to produce irreparable damage to the majority of the marrow cells. But much more information is necessary before any assessment can be made of the probable hazards of low radiation doses. In this context, and as far as man is concerned, two important sources of information will be, firstly, a study of the genetic constitution of human tumours and, in particular, human leukaemias, and, secondly, more intensive and more rigorous studies to refine the accuracy of the existing dose-response curves and to explore the region of doses lower than those so far studied.

In conclusion, therefore, and to return in particular to the problem of food and crop contamination by radioactive material, it may be said that the genetic hazard must be extremely small, because the strontium fixed in the skeleton cannot contribute any appreciable dose to the gonads, and there is no reason to believe that any change may be expected in expectation of life. As regards tumour induction, it cannot be denied that some lesions may occur, but with peacetime levels of contamination they are likely to be very few and insignificant, as far as leukaemia is concerned, in comparison with those that may occur from the widespread use of diagnostic radiology.

\section{REFERENCES}

Brown, W. M. C. (1958). Brit. med. Bull. 14, 168.

Brown, W. M. C. \& Doll, R. (1957). Spec. Rep. Ser. med. Res. Coun., Lond., no. 295.

Brown, W. M. C. \& Doll, R. (1958). Brit. med. F. ii, 18 I.

Jones, H. B. (1957). In The Nature of Radioactive Fall-out and its Effects on Man. Washington, D.C.: U.S. Government Printing Office.

Loutit, J. F. (1958). Communication to U.N. Conference on the Peaceful Uses of Atomic Energy, Geneva.

Muller, H. J. (1954). In Radiation Biology, Vol. I. [A. Hollaender, editor] New York: McGraw-Hill.

National Research Council (1956). The Biological Effects of Atomic Radiation. Washington: National Academy of Sciences.

Russell, W. L. (1958). Communication to U.N. Conference on the Peaceful Uses of Atomic Energy, Geneva.

Seltser, R. \& Sartwcll, P. E. (1958). F. Amer. med. Ass. I66, $5^{8} 5$.

Warren, S. (1956). F. Amer. med. Ass. 162, 464. 